\title{
REMOCIÓN DE CROMO (VI) A PARTIR DE AGUA SINTÉTICA A NIVEL DE LABORATORIO, MEDIANTE EL USO DE HIDRÓXIDOS DOBLES LAMINARES (HDL)
}

\author{
Erika Murgueitio*a, Willian Pinto $^{\mathrm{b}}$, José Landivar ${ }^{\mathrm{c}}$
}

\begin{abstract}
RESUMEN
Se sintetizó cuatro tipos de hidróxidos dobles laminares (HDL) con Al/Mg relación molar 4:1, empleando químicos grado analítico y técnico. Se realizó ensayos por lotes donde se determinó que la capacidad de adsorción de cromo (VI) es proporcional a la concentración inicial, para los cuatro adsorbentes $(\alpha=0,05 ; \mathrm{F}>\mathrm{F}$ crítico). También se determinó que es viable el uso de reactivos de grado técnico de menor costo en la síntesis, ya que los adsorbentes tienen similar nivel de eficiencia, tanto con agua sintética (agua destilada con iones competidores: $80 \mathrm{ppm}$ $\mathrm{HCO}_{3}^{-}, 100 \mathrm{ppm} \mathrm{Cl}-120 \mathrm{ppm} \mathrm{SO}_{4}=$ y concentraciones diferentes de $\mathrm{Cr}(\mathrm{VI})$ como con agua industrial. En ensayos de equilibrio en lotes con agua sintética se estableció hasta un $54 \%$ de adsorción; con agua industrial se obtuvo hasta un 19\%, los dos a los 120 minutos. El modelo de isoterma que se ajusta al comportamiento de adsorción es el de Freundlich, el cual indica una adsorción en multicapas. Las cinéticas de adsorción concuerdan con el modelo de pseudo primer orden, lo que supone una adsorción física y de intercambio iónico. En la columna de lecho fijo con agua sintética se logró remover hasta un $70 \%$ de cromo (VI). Al tratar el agua industrial disminuyó hasta un $98,4 \%$ y con ello se mantuvo los valores por debajo del límite permisible para descargas al alcantarillado. Los HDL disminuyen significativamente cromo (VI) del efluente de una industria de cromado de grifería, en una columna de lecho fijo a un $\mathrm{pH}$ de 2,8. Se recomienda el uso de HDL para tratar efluentes altamente contaminados y poder transferirlos a una matriz sólida, de fácil manejo para gestores ambientales.
\end{abstract}

Palabras clave: Hidróxido doble laminar, cromo (VI), adsorción, cinética, isoterma.

\section{CHROME VI REMOVAL FROM SYNTHETIC WATER LEVEL LABORATORY, USING LAYERED DOUBLE HYDROXIDES (HDL)}

\begin{abstract}
Four types of double layered hydroxides (HDL) were synthesized with $\mathrm{Al} / \mathrm{Mg}$ molar ratio of 4:1, using analytical and technical grade reagents. Batch essays were conducted in which it was determined that the adsorption capacity of chromium VI is proportional to the initial concentration, to the four adsorbents $(\alpha=0,05 ; \mathrm{F}>\mathrm{F}$ critical). It was also determined that it is viable the use of lower cost technical grade reagents for the synthesis, since the adsorbents have similar level of efficiency, both with sintetic water (distilled water with competing ions: 80 ppm $\mathrm{HCO}_{3}^{-}, 100 \mathrm{ppm} \mathrm{Cl}^{-}, 120 \mathrm{ppm} \mathrm{SO}_{4}=$ and different concentrations of $\mathrm{Cr} \mathrm{VI}$ ) and industrial water. In batch equilibrium tests with synthetic water it was established a $54 \%$ of adsorption; with industrial water about 19\% was obtained, both at 120 minutes. The isotherm model that fits the adsorption behavior is Freundlich which indicates a multilayer adsorption. Adsorption kinetics is consistent with the pseudo-first order model, which is a physical adsorption and ion exchange. In a fixed bed column with synthetic water $70 \%$ removal

a Carrera de Ing. Geográfica y del Medio Ambiente, Universidad de las Fuerzas Armadas -ESPE, *Av.E1 Progreso S/N, Sangolquí, Ecuador, esmurgueitio@espe.edu.ec

b Carrera de Ing. Biotecnología, Universidad de las Fuerzas Armadas -ESPE.

c Escuela Politécnica del Litoral, Guayaquil-Ecuador FIMCBOR


of chromium VI was achieved. When industrial water was treated the removal reached to $98.4 \%$ and thus the values remained below the permissible limit for discharge to drain. The using of HDL in a fixed bed column at $\mathrm{pH}$ 2,8 significantly decreases chromium VI from the effluent in chrome industry appliances. Is recommended the Use of HDL to treat highly polluted effluents and to transfer them to a solid matrix, easily manageable for environmental managers

Key words: Laminar double hydroxide, chromium VI, adsorption, kinetics, isotherm.

\section{INTRODUCCIÓN}

Según Katz \& Salem¹, el cromo es un elemento que, dependiendo de su forma química, puede ser un micronutriente esencial, como un agente carcinogénico. En su aplicación industrial, forma parte de curtientes, pigmentos y conservantes textiles, aleaciones, pinturas antiincrustantes, catalizadores, agentes anticorrosivos, lodos de perforación, baterías de alta temperatura, fungicidas, conservantes de madera, recubrimientos metálicos y electrogalvanizados ${ }^{2}$. El cromo (VI) es utilizado en la industria de galvanoplastia para el cromado de piezas metálicas y según el Ministerio del Ambiente ${ }^{3}$, se presenta ocasionalmente en sus efluentes. En la industria petrolera está usualmente presente en las descargas de las refinerías, junto a otros metales pesados. El cromo en el medio ambiente puede acumularse por causas naturales o antropogénicas y puede transferirse al ecosistema: aire, aguas superficiales, sedimentos, aguas subterráneas, suelos y seres vivos. La contaminación por cromo (VI) representa un grave peligro ya que ha sido catalogado en la lista A de elementos cancerígenos. En trabajadores, la inhalación de gases de cromo (VI) ha provocado cáncer del pulmón. De igual manera la ingestión mediante agua potable ha generado un aumento de tumores estomacales ${ }^{4}$. Para la remoción del cromo (y de otros metales mesados) existen tratamientos primarios y secundarios que distan mucho de ser considerados eficientes ${ }^{2}$, además de poseer rendimientos bajos pueden representar un costo elevado. Se ha desarrollado procesos tecnológicos específicos para la eliminación de los metales pesados de las aguas residuales ${ }^{5}$, pero su aplicación es altamente costosa y están muy poco generalizados. La literatura científica reporta que los HDL se han usado exitosamente en la reducción de $\mathrm{SOx}^{6}$, en colorantes como azul de metileno, rojo congo ${ }^{7}$. En la presente investigación se utiliza los HDL para la remoción de $\mathrm{Cr}$ (VI), en agua contaminada, mediante el proceso de intercambio iónico, el cual resulta ser un método aceptable, con la preparación de HDL con grados reactivo y técnico. Este estudio propone la aplicación de una técnica innovadora, entre los métodos de remoción de cromo (VI) se encuentra la adsorción. Esta puede llevarse a cabo utilizando los HDL, que en estado natural se denominan hidrotalcitas. Los hidróxidos dobles laminares, (o LDHs, acrónimo de Layered double hydroxides) son una familia de compuestos inorgánicos de estructura laminar, denominados arcillas aniónicas ${ }^{8}$, y conocidos como compuestos tipo hidrotalcita ${ }^{9}$. La composición de los HDL está representada por la fórmula general:

$$
\left[\mathrm{M}_{1-\mathrm{x}}^{\mathrm{II}} \mathrm{M}_{\mathrm{x}}^{\mathrm{III}}(\mathrm{OH})_{2}\right]\left[\mathrm{A}^{\mathrm{n}-}\right]_{\mathrm{x} / \mathrm{n}} \cdot \mathrm{z} \mathrm{H}_{2} \mathrm{O}
$$

Donde:

- $\quad \mathrm{A}^{\mathrm{n}-}$ es un anión con carga $\mathrm{n}$

- $\quad \mathrm{M}^{\mathrm{II}}$ y $\mathrm{M}^{\mathrm{III}}$ son metales di y trivalentes, respectivamente 
Existen diversos métodos de síntesis de HDL. Los más comunes son aquellos basados en la precipitación de las sales solubles de cationes divalentes y trivalentes o de precursores alcóxido u orgánicos, mediante una solución alcalina ${ }^{8}$. Se encuentra en la literatura innumerables trabajos de este método de dos formas diferentes: coprecipitación en $\mathrm{pH}$ constante y coprecipitación en $\mathrm{pH}$ variable ${ }^{10}$.

\section{Reactivos}

\section{PARTE EXPERIMENTAL}

Se empleó reactivos grado analítico ACS (American Chemical Society): sulfato de magnesio heptahidratado $\left(\mathrm{MgSO}_{4} .7 \mathrm{H}_{2} \mathrm{O}, 98 \%\right.$, Merck), sulfato de aluminio octadecahidratado $\left(\mathrm{Al}_{2}\left(\mathrm{SO}_{4}\right)_{3} \cdot 18 \mathrm{H}_{2} \mathrm{O}, 98 \%\right.$, Sigma Aldrich), hidróxido de sodio $(\mathrm{NaOH}, 98 \%$, Merck), carbonato de sodio $\left(\mathrm{Na}_{2} \mathrm{CO}_{3}, 98 \%\right)$, dicromato de potasio $\left(\mathrm{K}_{2} \mathrm{Cr}_{2} \mathrm{O}_{7}, 98 \%\right)$, cloruro de sodio $(\mathrm{NaCl}$, $98 \%$, Merck) y bicarbonato de sodio $\left(\mathrm{NaHCO}_{3}, 98 \%\right.$, Panreac). Los químicos grado técnico empleados fueron: sulfato de magnesio heptahidratado $\left(\mathrm{MgSO}_{4} .7 \mathrm{H}_{2} \mathrm{O}, 98 \%\right)$, sulfato de aluminio $\left(\mathrm{Al}_{2}\left(\mathrm{SO}_{4}\right)_{3}, 19 \%\right)$, hidróxido de sodio $(\mathrm{NaOH}, 98 \%)$ y carbonato de sodio $\left(\mathrm{Na}_{2} \mathrm{CO}_{3}\right.$, $98 \%)$.

\section{Síntesis de hidróxidos dobles laminares (HDL)}

Los HDL fueron preparados siguiendo el protocolo de Cumbal\&Murgueitio11, en el Centro de Investigaciones Científicas de la Universidad de la Fuerzas Armadas - ESPE (actualmente CENCINAT Centro de Nanociencia y Nanotecnología), el cual es una modificación de la técnica desarrollada por Reichle ${ }^{12}$.

Los HDL preparados fueron de grado reactivo HGR, HGT grado técnico, HGROM grado reactivo óxidos metálicos, HGTOM grado técnico óxidos metálicos.

Los químicos empleados fueron de grado ACS y técnico. En la síntesis se empleó una solución A de sulfato de magnesio y sulfato de aluminio en relación molar de 1:4 (Mg/Al), una solución $\mathrm{B}$ de carbonato de sodio $\left(\mathrm{Na}_{2} \mathrm{CO}_{3}\right)$ e hidróxido de sodio $(\mathrm{NaOH})$. Las soluciones A y B se vertieron simultáneamente sobre un lecho de $50 \mathrm{~mL}$ de agua destilada. La mezcla se mantuvo bajo vigorosa agitación a un $\mathrm{pH}$ entre 8-9,5, a $\mathrm{pH}$ constante. Para la síntesis del HDL grado reactivo se utilizó $100 \mathrm{~mL}$ de solución A, junto a $80 \mathrm{~mL}$ de solución B. En el caso del HDL grado técnico se empleó $100 \mathrm{~mL}$ de la solución A y $120 \mathrm{~mL}$ de la solución B. Posteriormente, se centrifugó y lavó con agua destilada tres veces, para luego secar a $100^{\circ} \mathrm{C}$ durante 24 horas. Se trituró y tamizó con una luz de malla de 1,16 micras para separar las partículas de menor tamaño. Se calcinó los precipitados a $500^{\circ} \mathrm{C}$ por 2 horas para formar los gránulos de HDL.

Para los HDL con óxidos metálicos se siguió el procedimiento dado por Cumbal\&Murgueitio ${ }^{11}$; se utilizó $30 \mathrm{~g}$ de gránulos de HDL, los cuales fueron sumergidos en un contenedor plástico de 1,0 L que contenía $500 \mathrm{~mL}$ de $\mathrm{FeCl}_{3} \cdot 6 \mathrm{H}_{2} \mathrm{O}$ al $10 \%(\mathrm{p} / \mathrm{v})$. El contenedor se colocó luego en un agitador rotativo a $40 \mathrm{rpm}$ durante 1 hora y los gránulos luego de ser extraídos del contenedor fueron lavados con agua desmineralizada y posteriormente sumergidos en $500 \mathrm{~mL}$ de una solución de $\mathrm{NaOH}$ al $5 \%(\mathrm{p} / \mathrm{v})$. Los átomos de Fe (III) se precipitaron en la superficie de los gránulos formando una cubierta de varias micras. Finalmente, los gránulos recubiertos con los óxidos de hierro fueron sometidos a sucesivos baños con agua desmineralizada para ajustar el $\mathrm{pH}$ a un valor alrededor de 7,0; ver figura 1 . 


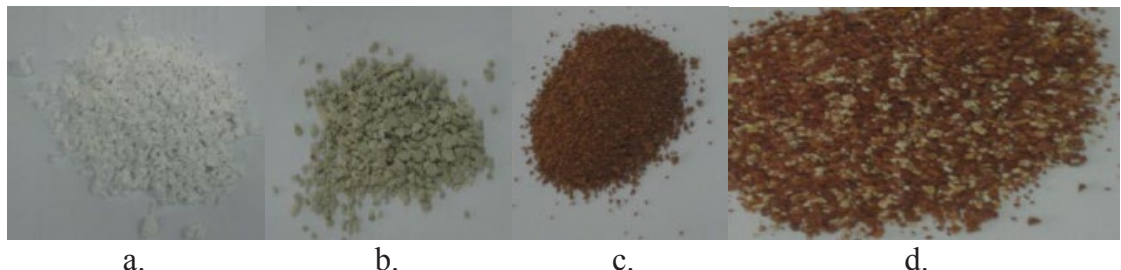

Figura 1. HDL: a) HGR grado reactivo, b) HGT grado técnico, c) HGROM grado reactivo óxidos metálicos, d) HGTOM grado técnico óxidos metálicos.

\section{Elaboración del agua sintética}

El agua sintética se preparó adicionando en agua destilada concentraciones diferentes de cromo (VI) empleando dicromato de potasio $\left(\mathrm{K}_{2} \mathrm{Cr}_{2} \mathrm{O}_{7}\right)$ y concentraciones de iones competidores de $80 \mathrm{mg} / \mathrm{L}$ de ion bicarbonato $\left(\mathrm{HCO}_{3}^{-}\right), 120 \mathrm{mg} / \mathrm{L}$ de ion sulfato $\left(\mathrm{SO}_{4}{ }^{-}\right)$y 100 $\mathrm{mg} / \mathrm{L}$ de ion cloruro $\left(\mathrm{Cl}^{-}\right)$.

\section{Análisis químico}

Las alícuotas tomadas para la cuantificación de cromo (VI) fueron analizadas en el espectrómetro de absorción atómica Perkin Elmer AAnalyst200. Se utilizó gas acetileno como combustible (flujo $3,3 \mathrm{~L} / \mathrm{min}$ ) y aire como oxidante (flujo $10 \mathrm{~L} / \mathrm{min}$ ). En el equipo se empleó una lámpara de cátodo hueco para detección de cromo a una longitud de onda de $359,9 \mathrm{~nm}$.

\section{Ensayo en lote con agua sintética e industrial}

Se realizó el ensayo por lotes a fin de determinar la remoción de cromo (VI) en concentración conocida aplicando los cuatro tipos de HDL. Se colocó 0,2 g de HDL con $200 \mathrm{~mL}$ de agua sintética de cromo (VI), estas concentraciones fueron de 10, 50, 80 y $120 \mathrm{mg} / \mathrm{L}$ y con medición de pH de las soluciones. Se agitó a $700 \mathrm{rpm}$ por $120 \mathrm{~min}$, tomándose alícuotas de las soluciones iniciales y finales para realizar las mediciones en el equipo de Absorción Atómica Perkin Elmer AA100.

El ensayo tuvo tres repeticiones para cada concentración de cromo (VI) con cada adsorbente. Con los datos obtenidos se determinó la capacidad de adsorción y el porcentaje de adsorción. Los datos recolectados se procesaron estadísticamente en el software InfoStat versión libre, mediante la prueba de Fisher. Se realizó igual procedimiento con el agua industrial, comparándose con los resultados del agua sintetizada.

\section{Ensayo cinético con agua sintética e industrial}

Se colocó $0,2 \mathrm{~g}$ de HDL en $200 \mathrm{~mL}$ de las soluciones en forma independiente en concentraciones de $10,50,80,120 \mathrm{mg} / \mathrm{L}$ de cromo VI y agua industrial, evaluándose por triplicado cada una, con medición de pH y agitación de $700 \mathrm{rpm}$. Se tomó alícuotas de la concentración inicial y cada 15 minutos, realizándose las lecturas en el espectrofotómetro de absorción atómica Perkin Elmer AA200. Se determinó las concentraciones residuales de cromo (VI) y las 
capacidades de adsorción. Se ajustó los datos a los modelos cinéticos linealizados de pseudo primer orden, pseudo segundo orden, orden cero, primer orden, segundo orden y tercer orden. Finalmente, se obtuvo los coeficientes de ajuste $\mathrm{r}^{2} \mathrm{y}$ las constantes cinéticas.

\section{Isotermas de adsorción con agua sintética e industrial}

Se determinó las capacidades de adsorción y las concentraciones de equilibrio, a partir de los datos de saturación obtenidos en el anterior ensayo. Se evaluó los cuatro tipos de HDL sintetizados por triplicado. Para interpretar un posible mecanismo de adsorción se analizó el ajuste al modelo de isotermas de Langmuir y Freundlich, a los resultados:

Ecuación linealizada de Langmuir a través de la gráfica $\mathrm{C}_{\mathrm{e}} / \mathrm{q}_{\mathrm{e}} \mathrm{vs}_{\mathrm{e}}$ :

$$
\frac{C e}{q e}=\frac{1}{q \max } C e+\frac{1}{q \max k L} \quad 1
$$

donde, $\mathrm{q}_{\text {máx }}$ es la retención máxima de cromo VI en el HDL $\left(\mathrm{mg}^{*} \mathrm{~g}^{-1}\right)$ y $\mathrm{k}_{\mathrm{L}}$ es la constante de Langmuir $\left(\mathrm{L}^{*} \mathrm{mg}^{-1}\right)$.

Ecuación linealizada de Freundlich:

$$
\ln q e=\frac{1}{n} \ln C e+\ln k_{F} \quad 2 .
$$

Donde, $\mathrm{k}_{\mathrm{F}}$ es la constante de Freundlich $\left(\mathrm{mg}^{*} \mathrm{~g}^{-1}\right)$ y $\mathrm{n}$ es el factor de heterogeneidad.

\section{Ensayo en columna de lecho fijo con agua sintética e industrial}

En una columna de vidrio pírex de $1,1 \mathrm{~cm}$ de diámetro interno y $30 \mathrm{~cm}$ de longitud, se colocó un lecho de HGR de 13,5 cm de altura. Luego se colocó una capa de $1 \mathrm{~cm}$ de altura de fibra de vidrio debajo y encima del adsorbente para la distribución uniforme de flujo. Se preparó una solución de cromo (VI) de $5 \mathrm{mg} / \mathrm{L}$ y se alimentó la columna mediante una bomba peristáltica, a un caudal de $1 \mathrm{~mL} / \mathrm{min}$. El tiempo de retención hidráulico en el lecho fue de 20 min. El efluente fue recogido en el colector de fracciones marca Eldex PN 1243., y el control de volumen para cada tubo fue fijado en $20 \mathrm{~mL}$. Las muestras para las mediciones fueron tomadas cada 3 a 5 tubos; se realizó igual procedimiento para el agua industrial.

\section{RESULTADOS Y DISCUSIÓN Rendimiento de la síntesis de hidróxidos dobles laminares (HDL)}

El rendimiento de la síntesis de HDL con químicos de grado técnico es del 18\% en peso, en el caso de la síntesis con químicos grado ACS se incrementa el rendimiento a $26 \%$ en peso, debido a la reducción de impurezas. La mayor pérdida de peso se da en los procesos de lavado y secado, donde se elimina mayormente agua. Por otra parte, el costo de los químicos grado técnico es $90 \%$ menor al costo de los químicos grado ACS. 


\section{Ensayo en lotes con agua sintética}

Los resultados obtenidos en el experimento en lotes denotan la dependencia de la capacidad de adsorción con la concentración inicial de la solución. Esta relación se evidencia en los cuatro tipos de HDL evaluados. En la tabla 1, se muestra las capacidades de adsorción tomando en cuenta las concentraciones iniciales y el tipo de HDL. Se observa, por ejemplo, que para el HGR la capacidad de adsorción aumenta conforme aumenta la concentración inicial.

Tabla 1. Capacidad de adsorción de los HDL, conforme aumenta la concentración inicial de cromo VI. Después de 120 minutos, empleando 0,2 g de adsorbente.

\begin{tabular}{|c|c|c|c|c|}
\hline HDL & $\begin{array}{c}\mathrm{Cr}(\mathrm{VI}) \\
10 \mathrm{mg} / \mathrm{L} \\
\mathrm{pH} \mathrm{7,9}\end{array}$ & $\begin{array}{c}\mathrm{Cr}(\mathrm{VI}) \\
50 \mathrm{mg} / \mathrm{L} \\
\mathrm{pH} \mathrm{7,1}\end{array}$ & $\begin{array}{c}\mathrm{Cr}(\mathrm{VI}) \\
80 \mathrm{mg} / \mathrm{L} \\
\mathrm{pH} \mathrm{6,7}\end{array}$ & $\begin{array}{c}\mathrm{Cr}(\mathrm{VI}) \\
120 \mathrm{mg} / \mathrm{L} \\
\mathrm{pH} \mathrm{6,3}\end{array}$ \\
\hline HGR & 1,81 & 12,35 & 18,43 & 24,88 \\
\hline HGT & 1,26 & 7,10 & 17,62 & 18,2 \\
\hline HGROM & 1,76 & 6,76 & 9,29 & 19,05 \\
\hline HGTOM & 2,02 & 9,40 & 10,56 & 15,00 \\
\hline
\end{tabular}

Los resultados obtenidos se corroboran con el análisis de varianza. Donde se comparó las capacidades de adsorción a cada concentración, para cada tipo de hidróxido. Y se estableció diferencias significativas $(\alpha=0,05 ; \mathrm{F}>\mathrm{F}$ crítico). En la figura 2 , se encuentra la capacidad de adsorción en función de la concentración inicial. Se evidencia una ligera superioridad de remoción del HGR, especialmente a mayores concentraciones.

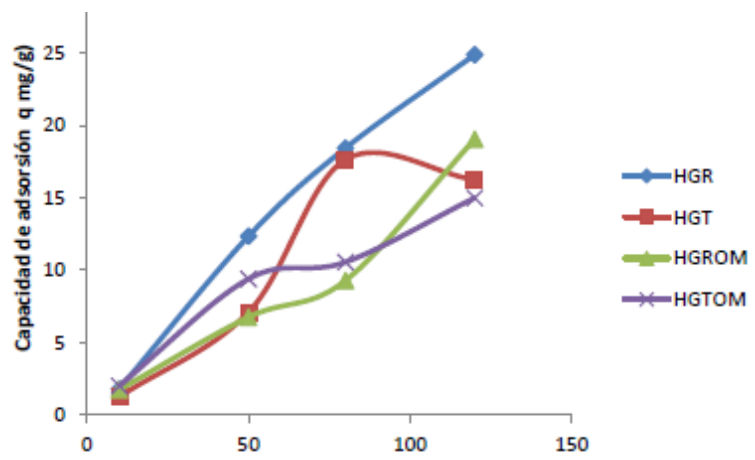

Figura 2. Adsorción de cromo VI sobre los HDL, a temperatura y presión constantes. HDL: HGR grado reactivo, HGT grado técnico, HGROM grado reactivo óxidos metálicos, HGTOM grado técnico óxidos metálicos. 
El experimento en lotes, para determinar la influencia del tipo de HDL en el porcentaje de adsorción, estableció que los adsorbentes presentan eficiencias similares. A concentraciones mayores de 50, 80 y $120 \mathrm{mg} / \mathrm{L}$, no existen diferencias significativas entre los $\operatorname{HDL}(\alpha=0,05$; $\mathrm{F}<\mathrm{F}$ crítico). Solamente el HGR presenta diferencias significativas $(\alpha=0,05 ; \mathrm{F}>\mathrm{F}$ crítico $) \mathrm{de}$ remoción a una concentración inicial de $10 \mathrm{mg} / \mathrm{L}$.

El pH de las soluciones fue de 6,3 a 7,9. En la tabla 2, se muestra los porcentajes de remoción para los cuatro HDL, a cuatro concentraciones de cromo VI.

Tabla 2. Porcentajes de adsorción de cada tipo de hidróxido laminar en diferentes concentraciones iniciales, después de 120 minutos.

\begin{tabular}{lllll}
\hline HDL & $\mathrm{Cr}(\mathrm{VI})$ & $\mathrm{Cr}(\mathrm{VI})$ & $\mathrm{Cr}(\mathrm{VI})$ & $\mathrm{Cr}(\mathrm{VI})$ \\
& $10 \mathrm{mg} / \mathrm{L}$ & $50 \mathrm{mg} / \mathrm{L}$ & $80 \mathrm{mg} / \mathrm{L}$ & $120 \mathrm{mg} / \mathrm{L}$ \\
& $\mathrm{pH} \mathrm{7,91}$ & $\mathrm{pH} 7,13$ & $\mathrm{pH} 6,71$ & $\mathrm{pH} 6,30$ \\
\hline HGR & $14,9 \%$ & $9,5 \%$ & $16,4 \%$ & $16,2 \%$ \\
HGT & $10,7 \%$ & $14,8 \%$ & $17,8 \%$ & $12,2 \%$ \\
HGROM & $8,5 \%$ & $14,5 \%$ & $10,3 \%$ & $14,2 \%$ \\
HGTOM & $6,4 \%$ & $16,4 \%$ & $12,0 \%$ & $10,9 \%$ \\
\hline
\end{tabular}

\section{Ensayo en lotes con agua industrial}

El efecto del tipo de HDL en los resultados del ensayo en lotes con agua industrial muestran que los porcentajes de adsorción son estadísticamente iguales $(\alpha=0,05 ; \mathrm{F}<\mathrm{F}$ crítico). El mejor porcentaje de remoción lo presenta el HGROM con 7,1\%, HGR con $6,7 \%$, HGT con $6,3 \%$ y HGTOM con $3,7 \%$.

\section{Ensayo de equilibrio con agua sintética}

En el ensayo de equilibrio se llegó a remover hasta un 54\% de cromo (VI), por parte del HGR a una concentración inicial de $10 \mathrm{mg} / \mathrm{L}$. El HGT adsorbió hasta un 47\% del contaminante, a una concentración de $10 \mathrm{mg} / \mathrm{L}$. El HGROM se saturó en 45\% a $80 \mathrm{mg} / \mathrm{L}$ y HGTOM al 41\% a $50 \mathrm{mg} / \mathrm{L}$.

\section{Ensayo de equilibrio con agua industrial}

En el análisis de equilibrio con agua industrial, se demuestra que la mayor remoción de $\mathrm{Cr}$ (VI) se obtuvo con el HGR con un 19,4\%, HGT con 17,8\%, HGTOM con 17,8\% y HGROM con $14,9 \%$. En la figura 3, se observa los porcentajes de adsorción dados por las alícuotas tomadas cada hora, para cada HDL. Conforme transcurre el tiempo el porcentaje de adsorción aumenta. Después de las 10 horas la adsorción deja de aumentar, indicando así el punto de saturación. No se realizó el análisis de remoción de de $\mathrm{Pb}$ y $\mathrm{Cu}$. 


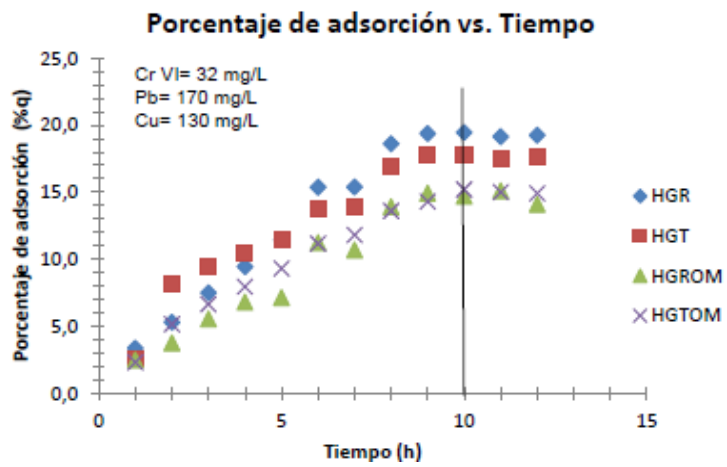

Figura 3. Historial de los porcentajes de adsorción de los hidróxidos laminares hasta el equilibrio.

\section{Isoterma de adsorción con agua sintética}

El modelo de isoterma que describe el proceso de adsorción de cromo (VI) para HGR, HGROM y HGTOM es el de Freundlich. En el caso del HGT se ajusta a la isoterma de Langmuir, ver tabla 3.

Tabla 3. Ajustes lineales a las ecuaciones linealizadas de Langmuir y Freundlich de los HDL.

\begin{tabular}{lcc}
\hline Tipo de HDL & $\begin{array}{c}\text { Isoterma de } \\
\text { Langmuir } \\
\left(\mathbf{r}^{\mathbf{2}}\right)\end{array}$ & $\begin{array}{c}\text { Isoterma de } \\
\text { Freundlich } \\
\left.\mathbf{( r}^{\mathbf{2}}\right)\end{array}$ \\
\hline HGR & 0,1853 & $\mathbf{0 , 9 5 5 9}$ \\
HGT & $\mathbf{0 , 9 9 9 0}$ & 0,9615 \\
HGROM & 0,4168 & $\mathbf{0 , 9 7 8 9}$ \\
HGTOM & 0,4168 & $\mathbf{0 , 9 9 6 4}$ \\
\hline
\end{tabular}




\section{Ensayo cinético con agua sintética}

Los resultados de la velocidad de adsorción de los HDL se ajustaron, en su mayoría, al modelo de capacidad de adsorción de pseudo primer orden, ver tabla 4.

Tabla 4. Ajustes de $\mathrm{r}^{2}$ para las ecuaciones cinéticas de los diferentes tipos de HDL a 120 minutos.

\begin{tabular}{cccccccc}
\hline HDL & $\begin{array}{c}\text { Cromo } \\
\text { VI } \\
(\mathbf{m g} / \mathbf{L})\end{array}$ & $\begin{array}{c}\text { Orden } \\
\text { cero }\end{array}$ & $\begin{array}{c}\text { Primer } \\
\text { orden }\end{array}$ & $\begin{array}{c}\text { Segundo } \\
\text { orden }\end{array}$ & $\begin{array}{c}\text { Tercer } \\
\text { orden }\end{array}$ & $\begin{array}{c}\text { Pseudo } \\
\text { primer } \\
\text { orden }\end{array}$ & $\begin{array}{c}\text { Pseudo } \\
\text { segundo } \\
\text { orden }\end{array}$ \\
\hline HGR & 10 & 0,9222 & 0,9273 & 0,9350 & $\mathbf{0 , 9 4 0 7}$ & $\mathbf{0 , 9 9 5}$ & 0,3612 \\
& 50 & 0,5045 & 0,5028 & 0,5027 & 0,759 & $\mathbf{0 , 6 3 0 8}$ & 0,5447 \\
& 80 & 0,9082 & 0,9086 & 0,9069 & 0,9044 & $\mathbf{0 , 9 3 6 4}$ & 0,0045 \\
HGT & 120 & 0,8143 & 0,8178 & 0,8185 & 0,8189 & $\mathbf{0 , 8 4 3 3}$ & 0,5966 \\
& 10 & 0,9595 & $\mathbf{0 , 9 6 1}$ & 0,9577 & 0,9574 & $\mathbf{0 , 9 7 5 2}$ & 0,0494 \\
& 50 & 0,8391 & 0,8485 & 0,8526 & 0,857 & $\mathbf{0 , 9 6 8 2}$ & 0,8948 \\
& 80 & 0,8261 & 0,8491 & 0,8598 & 0,8698 & $\mathbf{0 , 9 4 0 3}$ & 0,9499 \\
HGROM & 120 & 0,9274 & $; 0,9255$ & 0,93 & 0,8564 & 0,6777 & $\mathbf{0 , 9 4 8 4}$ \\
& 10 & 0,9260 & 0,9583 & 0,9625 & $\mathbf{0 , 9 6 6 4}$ & $\mathbf{0 , 9 7 4 8}$ & 0,8720 \\
& 50 & 0,7500 & 0,7538 & 0,7551 & 0,7566 & $\mathbf{0 , 8 6 3 2}$ & 0,6259 \\
& 80 & 0,6054 & 0,5895 & 0,5881 & 0,5866 & 0,9247 & $\mathbf{0 , 9 7 5 4}$ \\
& 120 & 0,8343 & 0,8548 & 0,8607 & 0,8668 & $\mathbf{0 , 9 9 0 5}$ & 0,9796 \\
& 10 & 0,9500 & 0,9512 & 0,9540 & $\mathbf{0 , 9 5 6 9}$ & $\mathbf{0 , 9 8 0 2}$ & 0,8125 \\
& 50 & 0,8866 & 0,9053 & 0,9084 & $\mathbf{0 , 9 1 1 2}$ & $\mathbf{0 , 9 2 6 8}$ & 0,7848 \\
& 80 & 0,7707 & 0,8211 & 0,8263 & 0,8316 & $\mathbf{0 , 9 8 3 5}$ & 0,9766 \\
& 120 & 0,7014 & 0,7297 & 0,7272 & 0,7241 & $\mathbf{0 , 7 6 7 7}$ & 0,3676 \\
\hline
\end{tabular}

\section{Ensayo cinético con agua industrial}

Los resultados de los ensayos cinéticos, realizados en el agua industrial contaminada con 32 ppm Cr (VI), con los HDL, se evidencian en la tabla 5, el cual es de pseudo primer orden para HGR y HGROM, y primer orden para HGT y HGTOM.

Tabla 5. Coeficientes de correlación $r^{2}$ de los modelos cinéticos empleados con agua industrial

\begin{tabular}{lcccccc}
\hline HDL & $\begin{array}{c}\text { Orden } \\
\text { cero }\end{array}$ & $\begin{array}{c}\text { Primer } \\
\text { orden }\end{array}$ & $\begin{array}{c}\text { Segundo } \\
\text { orden }\end{array}$ & $\begin{array}{c}\text { Tercer } \\
\text { orden }\end{array}$ & $\begin{array}{c}\text { Pseudo } \\
\text { primer } \\
\text { orden }\end{array}$ & $\begin{array}{c}\text { Pseudo } \\
\text { segundo } \\
\text { orden }\end{array}$ \\
\hline HGR & 0,8811 & 0,8538 & 0,8561 & 0,8564 & $\mathbf{0 , 9 6 1 6}$ & 0,906 \\
HGT & 0,8400 & $\mathbf{0 , 8 4 1 2}$ & 0,8377 & 0,8343 & 0,8170 & 0,7665 \\
HGROM & 0,3827 & 0,3851 & 0,3849 & 0,3834 & $\mathbf{0 , 9 3 6 7}$ & 0,9341 \\
HGTOM & 0,7410 & $\mathbf{0 , 7 4 3 4}$ & 0,7386 & 0,7352 & 0,6420 & 0,6258 \\
\hline
\end{tabular}




\section{Ensayo en columna de lecho fijo con agua sintética}

Se alcanzó una remoción del 70\% de Cr VI, al reducirse la concentración de $5 \mathrm{mg} / \mathrm{L}$ a 1,55 $\mathrm{mg} / \mathrm{L}$. La marcha se mantuvo hasta el equilibrio, el cual alcanzó a los 171 BV, a las 36 horas de operación. Se extrapoló la concentración máxima de remoción cuyo valor corresponde a 9,56 mg/L. En la figura 4 se observa la concentración normalizada de cromo VI del efluente de la columna. En los volúmenes de lecho inicial la concentración normalizada marca 0,31. Conforme aumentan los volúmenes de lecho la concentración se acerca al valor de 1 .

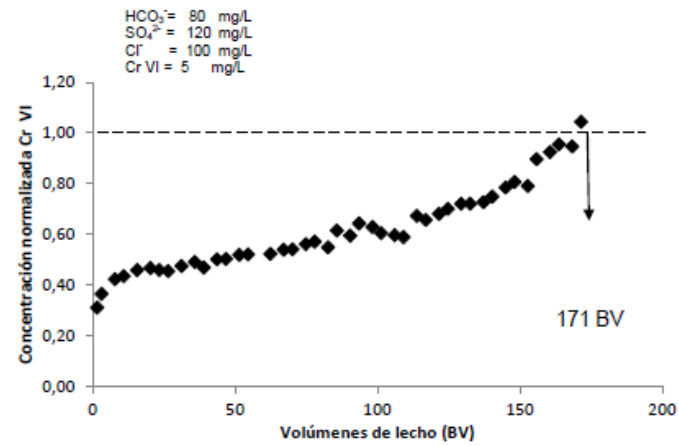

Figura 4. Concentración normalizada del efluente de la columna conforme pasan los volúmenes de lecho.

\section{Ensayo en columna de lecho fijo con agua industrial}

Con la remoción de Cr (VI), en el ensayo en columna de lecho fijo con agua industrial, proveniente del cromado de grifería, se obtuvo concentraciones en los efluentes bajo los límites máximos permitidos en la normativa ecuatoriana Texto Unificado de Legislación Ambiental Ecuatoriana (TULSMA), libro VI, anexo I , tabla $12(0,5 \mathrm{mg} / \mathrm{L}$ de $\mathrm{Cr}$ VI). El proceso se realizó hasta el volumen de lecho número 32.

\section{CONCLUSIONES}

- El ion cromato es removido, debido a la capacidad de intercambiar aniones de los HDL, que es una de sus principales propiedades. La reacción de intercambio aniónico es de tipo topotáctico, es decir, la estructura de las láminas se conserva, mientras que los enlaces más débiles aniones-láminas se rompen ${ }^{13}$.

- A pesar de que los iones cromatos desplazan con poca facilidad a los iones carbonatos, según el orden de preferencia de reemplazo de los iones14, sin embargo se obtuvo resultados de remoción de cromo (VI) de 54\% en agua sintética y en agua industrial de $19 \%$.

- En el ensayo de columna de lecho fijo con agua sintética removió un 70\% de cromo (VI), con agua industrial se obtuvo un $98,4 \%$ de remoción.

- La cinética de adsorción de cromo (VI) en HDL con agua sintética e industrial se ajustan al modelo de pseudo primer orden. No obstante los modelos lineales de velocidad de reacción no presentaron buenos ajustes.

- El modelo de isoterma que se ajusta al comportamiento de adsorción de los iones cromato $\left(\mathrm{CrO}_{4}{ }^{=}\right)$sobre la superficie de HGR, HGROM, HGTOM es el de Freundlich y para HGT 
es el de Langmuir.

- La utilización de HDL, grado técnico y reactivo, poseen un rendimiento de remoción de $\mathrm{Cr}$ (VI) apreciable, por tanto se podría utilizar los HDL grado técnico al poseer un costo mucho menor de fabricación.

\section{AGRADECIMIENTO}

Un agradecimiento especial al Centro de Nanociencia y Nanotecnología de la Universidad de las Fuerzas Armadas - ESPE y a su Director el PhD. Luis Cumbal, por su disposición a la apertura del centro para la realización de esta investigación.

\section{BIBLIOGRAFÍA}

1. S.A. Katz \& H. Salem, The biological and environmental chemistry of chromium. VCH, New York, 1994.

2. US.EPA, Environmental Protection Agency, Toxicological review of hexavalent chromium. (CAS No. 18540-29-9), Washington D.C U.S., 1998a

3. Ministerio del Ambiente, Información de plomo y cadmio en el Ecuador, Quito Ecuador, 2006.

4. Agencia para Sustancias Tóxicas y el Registro de Enfermedades, (ATSDR), Reseña Toxicológica del Cromo. Atlanta, U.S.A., 2008.

5. S.A.K. Palmer, T.J. Nunno, M.A. Breton, D.M. Sullivan, N.F. Surprenant. Metal cyanide containing wastes: Treatment technologies. Pollution Technology Review 158. Noyes Data Corporation, New Yersey, USA. 721 pp, 1988.

6. R. Cuevas, M.T. Bueno, J. Ramírez, C. Salcedo, B. Mar, F. Pedraza, Hidrotalcitas como precursores de óxidos de emisiones de SOx, Revista Mexicana de Ingeniería Química, 2003;2(1):1-7

7. J. Cavalheiro Rodriguez. Sintesis, Caracterização e Aplicações de argilas aniônicas tipo hidrotalcita, Universidad Federal do Rio Grande do Sul. Instituto de Química, Porto Alegre -Brasil, 2007.

8. S. Abelló. Síntesi i Caracterització de Materials Bàsics i la seva aplicación en reaccions d'interès industrial. Universit'E De Montpellier Ii Tarragona, 2005.

9. F. Cavani, F. Trifiró, A. Vaccari. Catalysis Today, 1991; 11(2):173

10. A. Roy, C. Forano, K. El Malki, J.P. Besse. Anionic Clays: Trends in Pillaring Chemistry; Occelli, M.L. and Robson, H.E.; Synthesis of Microporous Materials, New York, Van Nostrand Reinhold, 1992; 2:108-169.

11. L.Cumbal\&E.Murgueitio. Remoción de arsénico a nivel de laboratorio con sorbentes emergentes, Congreso Ciencia y Tecnología, Ecuador ISSN: 1390-4663, ESPE, June 6-8, 2012.

12. W.T. Reichle. Solid State Ionics, 1986; 44(17): 22,135

13. S. Miyata. Anion exchange properties of hydrotalcite-like compounds, Clays and Clay Minerals ,1983; 31: 305-311.

14. H. Olfs, L. Torres-Dorante, R. Eckelt, H. Kosslick. Comparison of different synthesis routes for $\mathrm{Mg}-\mathrm{Al}$ layered double hydroxides ( $\mathrm{LDH})$ : Characterization of the structural phases and anion exchange properties, Appl. Clay Sci., 2009; 3: 459-464. 